ONLINE TSST ASSESSED BY PHOTOPLETHYSMOGRAPHY

\title{
Induction of Acute Stress Through an Internet-Delivered Trier Social Stress Test as Assessed by Photoplethysmography on a Smartphone
}

\author{
Helen Harvie ${ }^{1}$, Barbie Jain ${ }^{1}$, Benjamin W. Nelson ${ }^{2}$, Erik L. Knight ${ }^{3}$, Leslie E. Roos ${ }^{1}$, and Ryan J. \\ Giuliano $^{1}$ \\ ${ }^{1}$ Department of Psychology, University of Manitoba \\ ${ }^{2}$ Department of Psychology, University of North Carolina, Chapel Hill \\ ${ }^{3}$ Department of Psychology and Neuroscience, University of Colorado Boulder
}

\begin{abstract}
Author Note
All data used in the present analyses is available at: https://osf.io/8qghs/. We have no conflict of interest to disclose.

Correspondence concerning this article should be addressed to Ryan J. Giuliano, Department of Psychology, University of Manitoba, Duff Roblin Bldg, 190 Dysart Rd, Winnipeg, MB, R3T 2N2, Canada. Email: ryan.giuliano@umanitoba.ca
\end{abstract}


ONLINE TSST ASSESSED BY PHOTOPLETHYSMOGRAPHY

\begin{abstract}
Recent studies have demonstrated the feasibility of administering the Trier Social Stress Test (TSST) through the internet, with major implications for promoting inclusivity in research participation. However, online TSST studies to date are limited by a lack of control groups and the need for biological measures of stress reactivity that can be fully implemented online. Here, we test smartphone-based photoplethysmography as a measure of heart rate reactivity to an online variant of the TSST. Results demonstrate significant acceleration in heart rate and heightened selfreported stress and anxiety in the TSST condition relative to a placebo version of the TSST. The placebo condition led to a significant increase in self-reported stress and anxiety relative to baseline levels, but this increase was smaller in magnitude than that observed in the TSST condition. These findings highlight the potential for smartphone-based photoplethysmography in internet-delivered studies of cardiac reactivity and demonstrate that it is critical to utilize random assignment to a control or stressor condition when administering acute stress online.
\end{abstract}

Keywords: acute stress, internet, Trier Social Stress Test, heart rate, photoplethysmography 
ONLINE TSST ASSESSED BY PHOTOPLETHYSMOGRAPHY

Induction of Acute Stress Through an Internet-Delivered Trier Social Stress Test as Assessed by Photoplethysmography on a Smartphone

Since its inception, the Trier Social Stress Test (TSST) has widely been regarded as the gold-standard method for inducing acute psychosocial stress, in terms of self-report and activation of neurobiological systems (Kirshbaum, Pirke, \& Hellhammer, 1993). Effect sizes of stress reactivity during the TSST are generally large for behavioural and biological measures (Goodman, Janson, \& Wolf, 2017), and have been replicated in children and adults, group formats (Von Dawans, Kirschbaum, \& Heinrichs, 2011), and virtual environments (e.g., Reim et al., 2020; Shiban et al., 2016). However, these TSST variants have all been limited to in-person laboratory testing.

The COVID-19 pandemic, and subsequent global shut down of in-person research, highlights the need for innovations in remote experimental assessments, which have the added potential of being more resource-friendly and inclusive (Kirschbaum, 2021). Recently, two studies have demonstrated the efficacy of internet-delivered variants of the TSST (iTSST) for children (Gunnar et al., 2021) and adults (Eagle, Rash, Tice, \& Proeschold-Bell, 2021) by demonstrating significant self-reported and neurobiological stress reactivity. However, both studies lacked a control group, limiting the ability to make inferences about the magnitude and timing of stress reactivity or determine whether significant reactivity had occurred over and above the inherent evaluative threat of being observed by researchers (Roos et al., 2017). Absence of a control group may be particularly problematic for studies of stress using a video chat interface, which may amplify elements of psychosocial stress due to a minimization of nonverbal cues and overemphasis on facial expressions (Sander \& Bauman, 2020). Here, we utilized 
ONLINE TSST ASSESSED BY PHOTOPLETHYSMOGRAPHY

a control group undergoing a placebo version of the TSST (Het et al., 2009) to control for any

elevations in stress that may be related to participating in a video chat mediated iTSST. When

considering the potential for iTSST to promote inclusive sampling practices (Kirschbaum, 2021), there is also a critical need to adapt biological stress reactivity protocols to be fully implemented online. Internet-delivered TSST research with salivary biomarkers such as cortisol (e.g., Gunnar et al., 2021) is only accessible to laboratories with the funding to cover the procurement, shipping, and processing of samples. ${ }^{1}$ Similar limitations exist for iTSST studies utilizing ambulatory cardiac equipment (e.g., Eagle et al., 2021), which require shipping and return shipping of relatively costly hardware that participants must be trained to use.

A primary aim of the present study was to test whether smartphone-delivered photoplethysmography (PPG) could serve as an accessible biological measure for online-variants of the TSST. Smartphone-delivered PPG uses a combination of the phone's flash and camera to measure changes in light intensity of subcutaneous tissue, which can be used as an index of heart rate with comparable reliability to electrocardiogram and pulse oximetry (De Ridder et al., 2018). In terms of increasing accessibility of research, free apps offering PPG indices of heart rate are currently available on the major smartphone operating systems and available to billions of consumers around the world (O'dea, 2020). Given that the TSST reliably leads to acceleration in heart rate due to sympathetic nervous system activation (Kim et al., 2018), we predicted that participants completing the TSST online would be able to self-administer PPG collection on their

\footnotetext{
${ }^{1}$ This is not to say that salivary collection off site is difficult for researchers with available funding, as the feasibility of salivary research by mail has been demonstrated in numerous studies of the cortisol awakening response, for example (see Boggero et al., 2017 for a review).
} 
ONLINE TSST ASSESSED BY PHOTOPLETHYSMOGRAPHY

own smartphones, and that these measures would be sufficiently reliable to capture TSST-related increases in a PPG index of heart rate.

\section{Methods}

\section{Participants}

Participants were students from a university in central Canada who participated in exchange for course credit for an introductory psychology course between September 2020 and April 2021. To be eligible for participation, individuals needed a working laptop and smartphone, to not be taking a medication known to accelerate heart rate (e.g., tricyclic antidepressants), to not have received a DSM-V recognized mental health diagnosis in the past 12 months, and to not have met clinical criteria for "severe" anxiety or depression symptoms. In order for these results to be comparable to a related study of stress buffering by peers and household pets, additional eligibility requirements included participants needing to have a peer available for a phone call or a household pet (i.e., dog or cat) available for a short interaction. ${ }^{2}$

One hundred and twenty participants who met the above eligibility criteria completed an internet-delivered variant of the iTSST or a placebo control condition. Sixteen $(13.3 \%)$ of those participants were excluded from analysis, either due to technical difficulties preventing heart rate data collection $(n=13)$, the occurrence of an interruption to the TSST procedures $(n=2)$, or non-compliance with self-report protocols $(n=1)$. The final sample of 104 participants $(n=50$, Stress; $n=54$, Control) consisted of 69 (66.3\%) females averaging approximately 20 years of age $(M=19.870, S D=4.22$; range, 17 to 45 years $)$. In terms of race and ethnicity, the sample was majority White/European (60.6\%), with representation from Asian (11.6\%), Filipino

\footnotetext{
${ }^{2}$ We note that two other conditions -- peer and pet buffering conditions --- were collected parallel to the present study and will be reported in future work. The present report is focused on proof of concept of the iTSST and the effects of the placebo version of the iTSST.
} 
ONLINE TSST ASSESSED BY PHOTOPLETHYSMOGRAPHY

$(11.5 \%)$, Indigenous $(8.7 \%)$, Other $(4.8 \%)$ and Black $(1.9 \%)$ communities (1 participant declined to respond). There were no significant differences in age $[t(102)=.340, p=.734]$ or gender $[t(102)=1.352, p=.179]$ between the Stress and Control groups. A minority of the female participants in this sample reported currently taking a hormonal contraceptive (36.2\%), which has previously been associated with altered stress reactivity as measured by cortisol but does not seem to introduce systematic bias into gender comparisons of cardiac reactivity (Lovallo et al., 2019; Schallmayer \& Hughes, 2010).

\section{Procedure}

All procedures were approved by the research ethics board of the corresponding author. In order to establish eligibility for the TSST protocol, volunteers were required to complete a battery of questionnaires before participating. These surveys were completed either on a day before the experiment $(n=83)$ or immediately before the experiment $(n=41)^{3}$.

Participants were randomly assigned to a Control (placebo) or Stressor condition before the date of their participation. Participants chose the time that they participated in the experiment, with available time slots between 09:00 am and 5:30 pm. When signing up for a session, participants were informed that they would receive an automated email 10 minutes before their scheduled time slot containing a hyperlink to a Zoom video chat. Upon arriving in the Zoom call, the participant was greeted by a researcher who administered informed consent, then established that the participant was alone and in a private space in their home and allowed the participant to ready themselves for the experiment. Participants were explicitly asked to check in with anyone who may be arriving at their residence during the experiment in order to

\footnotetext{
${ }^{3}$ There were no significant differences in self-reported stress and anxiety (all $p \mathrm{~s}>.33$ ) or heart rate (all $p \mathrm{~s}$ $>.25$ ) at any of the seven measurement timepoints between individuals who completed the questionnaires on a previous day and individuals who completed the surveys immediately before the experiment.
} 
ONLINE TSST ASSESSED BY PHOTOPLETHYSMOGRAPHY

ensure that participants remained in a private space with minimal interruption, to gather writing material, and to refrain from eating or drinking anything but water during the experiment.

The session then began with participants being asked to enable and share their screen throughout the Zoom session, in order to ensure compliance with the task protocol. Then participants were guided through downloading the Heart Rate Plus app and collecting an initial heart rate and VAS measurement. Immediately afterwards, participants were sent a YouTube link to watch a 5-minute video featuring scenes of underwater wildlife accompanied by tranquil instrumental music. Participants were instructed to be mindful of relaxing their body while watching the baseline video, to turn the audio on to a comfortable volume, and to not touch their computer until the video was complete. Immediately after the video was over, participants were then instructed to record a second heart rate and VAS measurement. Following this, all participants completed a 5-minute period of reading neutral articles from magazines or scientific journals. Each participant was sent the same six PDF files over the Zoom chat function, featuring articles chosen for being interesting but not provocative or emphasizing affective content, with participants allowed to read as many of the articles as they chose. While participants were reading, the researcher turned off their video and microphone feed but continued to monitor the activity of the participant to ensure task compliance. At the end of this 5-minute period, participants were asked to record a third heart rate and VAS measurement.

Participants then began either the iTSST or a placebo version of the iTSST adapted from Het and colleagues (2009) to control for general reactivity to performing speech and arithmetic. All participants, regardless of the assigned condition, were initially given the same instructions at the start of this task. Participants were told that they were to imagine that they were applying for a job, and that they had five minutes to prepare a five-minute speech. Their speech was to outline 
ONLINE TSST ASSESSED BY PHOTOPLETHYSMOGRAPHY

their strengths as an employee, how they overcome their weaknesses, and why they are the ideal employee. Participants were encouraged to use the five minutes allocated to preparation to take notes on their speaking points. The researcher shut off their video feed and microphone during this time and did not interact with the participant until the five-minute preparation period was complete.

Upon the completion of the speech preparation, participants assigned to the Stress condition were informed by the researcher that colleagues would be joining to watch their speech and to run the next few tasks. At this point, two panelists were admitted to the Zoom call from a waiting room, where they had been waiting in preparation. The researcher turned off their video and microphone feed, while one TSST panelist then outlined the expectations of the speech to the participant. The participant was to destroy or put away the notes they had prepared for the speech, and to set their Zoom screen to "Gallery" view in order to see each panelist clearly for the duration of the speech. Finally, the participant was instructed that they were to begin their speech and must continue talking throughout the 5-minute period. Throughout the speech, the panelists maintained neutral facial expressions and refrained from giving positive feedback to the participant. If the participant paused for a minimum of 3 seconds, the panelists interjected with phrases such as, "You still have time remaining," and, "Continue your speech."

Immediately upon completion of the speech, one of the panelists instructed the participant to complete a fourth heart rate and VAS measurement. Participants assigned to the Control condition also completed a 5-minute speech, but they did so without any researchers present and without the introduction of novel, unfamiliar panelists. The researcher informed Control participants that they could refer to the notes that they had made during the preceding preparation phase, outlined to the participant that they would be required to speak for a total of 
ONLINE TSST ASSESSED BY PHOTOPLETHYSMOGRAPHY

five minutes, and added that participants could discuss their coursework or the events of their day if their speech did not span the full five minutes. Then the researcher turned their video feed and microphone off during the speech. The researcher turned their video and microphone feed on after the five-minute period was complete to administer the fourth heart rate and VAS measurement.

Participants then began the arithmetic portion of the TSST task. For individuals assigned to the Stress condition, one of the panelists instructed the participant that they were to perform serial subtraction out loud from 2043 in increments of 17 as quickly and accurately as possible. If the participant made a mistake during this task, the panelists would intervene and ask the participant to start from the beginning. If the participant paused for any length of time, the panelists interjected with phrases such as, "You need to go as fast as you can," or, "Try not to pause between numbers during this task." Participants continued until the 5-minute period was complete, at which point one of the panelists instructed the participant to complete the fifth heart rate and VAS measurement. Upon completion of these measurements, the original researcher reactivated their video feed and microphone, and asked the panelists to leave the call. Individuals assigned to the Control condition also completed verbal arithmetic for five minutes, but as with the placebo speech task, they did so without any researchers present and without the introduction of unfamiliar panelists. Participants were also given an easier arithmetic task, to count up from zero in increments of 15 . The researcher turned off their video feed and microphone during the 5-minute period, turning their video feed and microphone back on at the end of this time to instruct the participant to complete a fifth heart rate and VAS measurement.

From this point on, all participants completed identical procedures regardless of the condition they were assigned to. Participants completed another 5-minute period reading 
ONLINE TSST ASSESSED BY PHOTOPLETHYSMOGRAPHY

magazine and news articles, while the researcher shut off their video feed and microphone so as not to interrupt. Immediately after the 5-minute reading period, participants completed a sixth heart rate and VAS measurement. Participants were then sent a YouTube link to watch a final 5minute baseline video, similar in content to the initial baseline video and with similar instructions for participants to relax. Upon completion of this 5-minute video, participants were asked to record a seventh heart rate and VAS measurement. Participants were then debriefed on their participation of the study specific to the condition they were assigned and were awarded their course credits.

\section{Measures}

Photoplethysmography (PPG) Index of Heart Rate. Heart rate was collected via photoplethysmography (PPG) using the Heart Rate Plus application (PVD Apps, 2015). Heart Rate Plus is freely available to download for iOS and Android mobile and tablet users, allowing participants to self-administered heart rate measures remotely in this study. This application implements photoplethysmography via a combination of light emission from the smartphone's flash and detection of light intensity in subcutaneous tissue (i.e., fingertip) by the smartphone's camera, allowing for the measurement and estimation of vital signals such as heart rate.

Measurements with the Heart Rate Plus app take approximately 30 seconds to three minutes and provide a single estimate of average heart rate during measurement. Participants were provided with verbal instructions along with a visual aid specific to the model of smartphone they were operating, guiding individuals to place their finger over the appropriate area of the camera lens(es) and flash of that particular model of smartphone, to sit as still as possible, and to refrain from talking during measurement. Heart rate measurements were collected at seven time points 
ONLINE TSST ASSESSED BY PHOTOPLETHYSMOGRAPHY

during the experimental session: 1) before a 5-minute resting baseline period at the beginning of the visit, 2) after the 5-minute resting baseline, 3) before the TSST or placebo condition, 4) immediately after the speech portion of the TSST, 5) immediately after the arithmetic portion of the TSST, 6) after a subsequent 5-minute recovery period of reading and immediately before a 5minute recovery baseline period, and 7) after the 5-minute recovery baseline. Each measure of heart rate was verbally communicated to the researcher during the session and entered into an online form sent to the participant via Qualtrics, with researchers confirming correct heart rate data entry after the session.

Visual Analogue Scales (VAS) of Perceived Stress and Anxiety. Immediately after completing each heart rate measurement, participants completed three Visual Analogue Scales (VAS) assessing “How ___ are you currently feeling?” for the terms "anxious", "stress", and “insecure" (Lesage, Berjot, \& Deschamps, 2012). Each VAS was administered with a sliding scale from 0 to 100, where 0 is not feeling anxious, stressed or insecure and 100 is feeling very anxious, stressed or insecure. At each time point, we averaged VAS measures of stress and anxiety to compute a single index of "stress and anxiety".

Mental Health Questionnaires. To establish eligibility for the experiment, participants completed a series of mental health questionnaires. The 7-item Generalized Anxiety Disorder Inventory (GAD-7; Spitzer et al., 2006) was utilized to determine whether participants met clinical criteria for severe anxiety. The GAD-7 uses a 4-point scale ranging from "not at all" to "nearly every day" to assess how often a participant may have experienced anxious feelings over the last two weeks, with clinical criteria for severe symptomatology represented by scores of 15 and higher. The 21-item Beck Depression Inventory II (BDI; Beck, Steer, \& Brown, 1996) was 
ONLINE TSST ASSESSED BY PHOTOPLETHYSMOGRAPHY

utilized to determine whether participants met clinical criteria for severe depression. The BDI assesses the frequency of participants' depressive symptoms over the past two weeks using a 4point scale ranging from low to high frequency of depressive symptomatology, with clinical criteria for severe depression represented by BDI scores of 30 and higher. We also created a brief questionnaire for participants to self-report any diagnoses of the following psychological disorders from the DMS-V: major depressive disorder, bipolar disorder, panic disorder, social anxiety disorder, generalized anxiety disorder, post-traumatic stress disorder, anorexia nervosa, bulimia nervosa, binge eating disorder, schizophrenia, attention deficit hyperactivity disorder, and substance use disorder. Participants were presented with a list of each disorder and asked to report whether they had received a diagnosis or treatment for that disorder a) in the past month, b) in the past 2 to 12 months, c) $1+$ years ago, or d) never.

\section{Analytic Plan}

Before primary models were examined, outliers in heart rate and self-reported data were winsorized to within 3 standard deviations of the means ${ }^{4}$. Subsequent heart rate and VAS averages of stress and anxiety were analyzed at each time point using a 2 (Group) x 7 (Time) repeated measures ANCOVA. Significant Group x Time interactions were followed up with Bonferroni-corrected pairwise comparisons. Given our hypothesis that the TSST manipulation would induce accelerations in heart rate and elevations in self-reported stress and anxiety, we expected pairwise comparisons of Stressor vs. Control measures to be significantly different during the TSST procedures. We were also interested in whether reactivity effects to the placebo procedures would be observed in the Control group, which would be reflected by pairwise

\footnotetext{
${ }^{4} \mathrm{R}$ scripts demonstrating outliers and effects of winsorization are available at: https://osf.io/8qghs/
} 
ONLINE TSST ASSESSED BY PHOTOPLETHYSMOGRAPHY

comparisons across timepoint, particularly for pre-TSST measures (e.g., timepoint 3) relative to during (i.e., timepoint 4) and after the TSST (i.e., timepoint 5). Gender was included as a covariate in the ANCOVA, given previous findings of gender differences in self-reported and cardiac reactivity to the TSST (Hamidovic et al., 2020). Significant effects of gender in the repeated measures ANCOVA were followed up with independent-samples $t$-tests comparing males and females.

\section{Results}

Descriptive statistics are shown in Table 1 for heart rate and self-reported stress and anxiety across the seven timepoints of the assessment. Table 2 presents the results of a Group (2) x Time (7) repeated measures ANCOVA performed on heart rate and combined stress and anxiety self-reports, controlling for participant gender.

There was a main effect of Group on heart rate $\left[F(1,101)=6.491, p=.012, \eta^{2}\right.$ partial $=$ $.060]$ and self-reported stress and anxiety $\left[F(1,101)=5.089, p=.026, \eta^{2}\right.$ partial $\left.=.048\right]$ such that the Control group demonstrated slower heart rate and lower ratings of stress and anxiety. However, these main effect were qualified by significant Group x Time interactions for both heart rate $\left[F(4.778,482.588)=2.449, p=.035, \eta^{2}\right.$ partial $\left.=.024\right]$ and self-reported stress and anxiety $\left[F(3.108,313.915)=7.960, p<.001, \eta^{2}\right.$ partial $\left.=.073\right]$. As shown in Figure 1 , heart rate acceleration and elevated ratings of stress and anxiety were observed in the Stress group relative to the placebo Control group beginning at the speech preparation phase (timepoint 3) and peaking during the TSST procedures (timepoints 4 and 5), with some continued separation between groups through the end of the visit. Follow-up pairwise comparisons with Bonferroni corrections confirmed significant elevations in heart rate for the Stressor group at timepoint 3 $\left[F(1,101)=5.020, p=.027, \eta^{2}\right.$ partial $\left.=.047\right]$, timepoint $4\left[F(1,101)=5.710, p=.019, \eta^{2}\right.$ partial $=$ 
ONLINE TSST ASSESSED BY PHOTOPLETHYSMOGRAPHY

$.054]$, timepoint $5\left[F(1,101)=6.652, p=.011, \eta^{2}\right.$ partial $\left.=.062\right]$, and timepoint $7[F(1,101)$

$=5.891, p=.017, \eta^{2}$ partial $\left.=.055\right]$; significant elevations in self-reported stress and anxiety for the

Stressor group were observed at timepoint $4\left[F(1,101)=13.092, p<.001, \eta^{2}\right.$ partial $\left.=.115\right]$,

timepoint $5\left[F(1,101)=13.913, p<.001, \eta^{2}\right.$ partial $\left.=.121\right]$, timepoint $6[F(1,101)=5.998, p=$

$.016, \eta^{2}$ partial $\left.=.056\right]$, and timepoint $7\left[F(1,101)=4.725, p=.032, \eta^{2}\right.$ partial $\left.=.045\right]$.

Self-reported stress and anxiety showed some reactivity to the placebo TSST procedures. Following up the significant Group x Time interaction, Bonferroni-corrected pairwise comparisons across timepoint were examined for heart rate and self-report in the Control group. As shown in Table 3, the Control group showed significant increases in self-reported stress and anxiety to the placebo procedures at timepoint 4 relative to timepoint $3\left[M_{\text {timepoint }} 4\right.$ - timepoint $3=$ 18.026, $S E=2.994, p<.001]$ and at timepoint 5 relative to timepoint $3\left[M_{\text {timepoint } 5}-\right.$ timepoint $3=$ 12.531, $S E=3.507, p=.011]$, although the magnitude of Control group reactivity at this

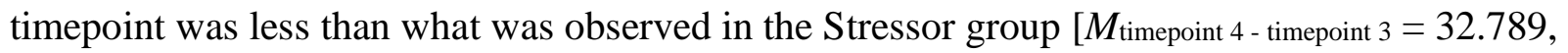
$S E=2.881, p<.001 ; M$ timepoint 5 - timepoint $3=29.506, S E=3.375, p<.001]$, as reported above. No significant differences in heart rate were observed across any of the measurement timepoints for the Control group (see Table 4). In contrast, the Stressor group showed significant elevations in

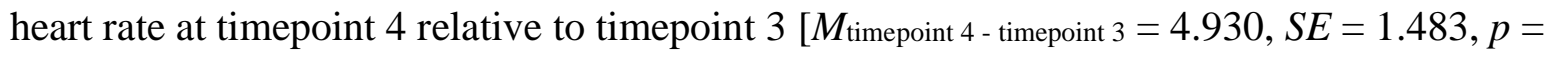
$.026]$.

Gender was a significant predictor of self-reported stress and anxiety throughout the assessment, as revealed by a main effect of $\operatorname{Gender}\left[F(1,101)=5.392, p=.022, \eta^{2}\right.$ partial $\left.=.051\right]$ and a Gender $\times$ Time interaction $\left[F(6,606)=3.161, p=.005, \eta^{2}\right.$ partial $\left.=.030\right]$. We followed this up with independent samples $t$-tests of all seven self-report measures of stress and anxiety (see Table 5). Female participants generally reported higher levels of stress and anxiety, but this was 
ONLINE TSST ASSESSED BY PHOTOPLETHYSMOGRAPHY

carried by elevated stress and anxiety at timepoint $4[t(49)=-2.239, p=.030]$ and timepoint 5 $[t(49)=-2.288, p=.026]$ independent of stress condition. No significant differences in heart rate as a function of Gender were observed.

\section{Discussion}

The aim of this study was to assess whether acute psychosocial stress could be induced by an internet-delivered variant of the Trier Social Stress Test (iTSST), and to establish whether photoplethysmography (PPG) measures of heart rate would be sufficient to detect cardiac reactivity to the iTSST. An additional aim was to examine the extent to which any observed stress reactivity associated with the iTSST differed from a placebo iTSST that controlled for task demands while minimizing the presence of additional psychosocial stressors. The results support our primary hypotheses: individuals who completed the iTSST showed significant elevations in heart rate and self-reported stress and anxiety to the iTSST procedures. Heart rate and selfreported stress and anxiety remained elevated relative to a Control group through the end of the assessment. We also observed significant reactivity to the placebo procedures in a Control group for self-reported stress and anxiety, with the iTSST group demonstrating reactivity over and above the Control group in terms of both heart rate and self-report. Taken together, these results replicate recent iTSST findings (Eagle et al., 2021; Gunnar et al., 2021) and extend those findings to demonstrate the feasibility of measuring perceived and biological stress reactivity entirely through internet-mediated platforms, while highlighting the necessity of a placebo condition to control for potential psychosocial stressors inherent to video chat assessments.

Comparisons of stress reactivity between groups demonstrate elevations in heart rate and self-reported stress and anxiety induced by the iTSST relative to placebo conditions. For heart rate, these differences onset at the anticipation stage immediately before speech preparation 
ONLINE TSST ASSESSED BY PHOTOPLETHYSMOGRAPHY

(timepoint 3) and remained elevated relative to the Control group throughout the end of the visit. However, when considering reactivity within group, the Stressor group only showed elevated heart rate at the measurement taking during the iTSST (timepoint 4) relative to their pre-iTSST heart rate. Future research is needed to determine the extent to which the iTSST leads to lasting cardiac acceleration beyond the iTSST procedures. One important direction for this research is to explore whether there are individual differences in the timing and degree to which participants show cardiac recovery from the iTSST. Based on the body of literature demonstrating the potential for social inputs to minimize the impact of acute stress reactivity (e.g., Hostinar, Sullivan, \& Gunnar, 2014), it is likely that social supports from family, peers, and even household pets are an important predictor of cardiac recovery from the iTSST. Future work in our lab will be examining how such social inputs before and after the iTSST may impact perceived and biological reactivity to psychosocial stress.

A major innovation of this study was the utilization of participant self-administered measures of heart rate implemented with each participant's own smartphone. To date, experimental studies of biological reactivity to stress performed in participants' homes has involved transporting some amount of laboratory equipment or materials from the laboratory, to participants' homes, and back to the laboratory (DeJoseph et al., 2019; Eagle et al., 2021; Gunnar et al., 2021). Here, we demonstrate the possibility of measuring cardiac stress reactivity in any adult with internet access, a smartphone, and the cognitive capacity to engage in research activities. The implications are nearly open-ended and raise the specter of an international study of stress reactivity. By transcending traditional geographical boundaries, representative samples can theoretically be reached around the world, perhaps most importantly improving the representation of racial, ethnic, and other marginalized groups. Boundaries for inclusion in 
ONLINE TSST ASSESSED BY PHOTOPLETHYSMOGRAPHY

research are not only reduced for participants, but for scientists themselves, through the use of a freely available, lo-fi index of heart rate. We expect the utility of smartphone-based PPG to extend to studies of cardiac activity and behavior that do not involve the TSST.

Consistent with previous findings from the TSST administered in laboratories (e.g., Kelly et al., 2008), we found robust gender differences in self-reported reactivity to the iTSST but no gender differences in cardiac reactivity. Females reported greater stress and anxiety than males during and immediately after the iTSST, as well as during and immediately after the placebo version of the iTSST. As has been discussed by others, such a pattern of results could be due to a number of factors including sex differences in neuroendocrine responsivity (Allen et al., 2014) and the socialization of emotional expression and awareness (Dedovic, Wadiwalla, Engert, \& Pruessner, 2009).

A potential limitation to this study is its occurrence during the COVID-19 pandemic, given evidence that stress and anxiety levels have been elevated relative to historical averages (e.g., Cameron et al., 2020). However, we anticipated this limitation and chose to randomly assign participants to a comparison placebo condition, in order to control for any influence of COVID-19 related stressors on stress reactivity. Still, it is possible that the stress reactivity observed here is atypical relative to what might be observed at other moments in history. This limitation is particularly problematic for extant studies of iTSST variants that did not employ a control group (i.e., Eagle et al., 2021; Gunnar et al., 2021), raising questions about the extent to which observed stress reactivity was specific to the iTSST procedures. Replication of iTSST studies may be necessary in the future, when humans have had the time to process and potentially recover from the psychological effects of the COVID-19 pandemic.

The influence of environmental and household distractors served to be somewhat of an 
ONLINE TSST ASSESSED BY PHOTOPLETHYSMOGRAPHY

uncontrollable source of variance in this study. There were subtle differences in individual assessments, such as family members or roommates disrupting the participants during the assessments, participants spontaneously turning their cameras off and not being visible for short periods of time, and internet connectivity issues. Although we excluded two participants from analyses who experienced significant interruptions during their assessment, this does not account for the less noticeable, transient interruptions that may occur at any point during a video interaction over the internet. We have recently begun video and audio analysis of the recorded iTSST sessions in order to quantify the variety of environmental factors that may explain some variance in the measures reported here.

Another limitation is the potential influence of skin tone on peripheral measures of heart rate (Nelson et al., 2020). Although evidence is mixed, studies suggest an association between darker skin tone and less accurate optical measurements of heart rate (Weiler et al., 2017). Yet, a majority of studies do not report or measure skin tone. When considering the potential for smartphone-based PPG to promote diversity and inclusivity in studies of heart rate, future studies would be well-poised to address how skin tone may covary with accuracy of optical heart rate measures such as PPG.

\section{Conclusion}

The current study demonstrated a low-cost and user-friendly internet variant of the TSST, which could be ideal for researchers attempting to study acute stress in hard to access populations. Future research is needed to extend this study to a larger, international sample in order to examine racial, ethnic, and cultural differences that may exist in stress reactivity. There is an additional need for future research examining potential sources of buffering from stress reactivity, such as talking or interacting with a close peer, or having social support at home 
ONLINE TSST ASSESSED BY PHOTOPLETHYSMOGRAPHY

during the assessment. The protocol employed here could also be utilized to evaluate the efficacy of other forms of stress reduction (e.g., mindfulness-based stress reduction). Taken together, the induction of psychosocial stress reactivity over the internet holds much promise for studies of stress and coping. 
ONLINE TSST ASSESSED BY PHOTOPLETHYSMOGRAPHY

\section{References}

Allen, A. P., Kennedy, P. J., Cryan, J. F., Dinan, T. G., \& Clarke, G. (2014). Biological and psychological markers of stress in humans: Focus on the Trier Social Stress Test. Neuroscience \& Biobehavioral Reviews, 38, 94-124.

Beck, A. T., Steer, R. A., \& Brown, G. (1996). Beck depression inventory-II. Psychological Assessment.

Boggero, I. A., Hostinar, C. E., Haak, E. A., Murphy, M. L., \& Segerstrom, S. C. (2017). Psychosocial functioning and the cortisol awakening response: Meta-analysis, P-curve analysis, and evaluation of the evidential value in existing studies. Biological psychology, 129, 207-230.

Cameron, E. E., Joyce, K. M., Delaquis, C. P., Reynolds, K., Protudjer, J. L., \& Roos, L. E. (2020). Maternal psychological distress \& mental health service use during the COVID19 pandemic. Journal of Affective Disorders, 276, 765-774.

Dedovic, K., Wadiwalla, M., Engert, V., \& Pruessner, J. C. (2009). The role of sex and gender socialization in stress reactivity. Developmental psychology, 45(1), 45.

DeJoseph, M. L., Finegood, E., Raver, C., \& Blair, C. B. (2019). Measuring stress reactivity in the home: Preliminary findings from a version of the Trier Social Stress Test (TSST-H) appropriate for field-based research. PsyArxiv. https://doi.org/10.31234/osf.io/5qapw

De Ridder, B., Van Rompaey, B., Kampen, J. K., Haine, S., \& Dilles, T. (2018). Smartphone apps using photoplethysmography for heart rate monitoring: meta-analysis. JMIR cardio, 2(1), e4.

Eagle, D. E., Rash, J. A., Tice, L., \& Proeschold-Bell, R. J. (2021). Evaluation of a remote, internet-delivered version of the Trier Social Stress Test. International Journal of 
ONLINE TSST ASSESSED BY PHOTOPLETHYSMOGRAPHY

Psychophysiology.

Gunnar, M. R., Reid, B. M., Donzella, B., Miller, Z. R., Gardow, S., Tsakonas, N. C., ... \&

Bendezú, J. J. (2021). Validation of an online version of the Trier Social Stress Test in a study of adolescents. Psychoneuroendocrinology, 125, 105111.

Hamidovic, A., Van Hedger, K., Choi, S. H., Flowers, S., Wardle, M., \& Childs, E. (2020).

Quantitative meta-analysis of heart rate variability finds reduced parasympathetic cardiac tone in women compared to men during laboratory-based social stress. Neuroscience \& Biobehavioral Reviews.

Het, S., Rohleder, N., Schoofs, D., Kirschbaum, C., \& Wolf, O. T. (2009). Neuroendocrine and psychometric evaluation of a placebo version of the 'Trier Social Stress Test'. Psychoneuroendocrinology, 34(7), 1075-1086.

Hostinar, C. E., Sullivan, R. M., \& Gunnar, M. R. (2014). Psychobiological mechanisms underlying the social buffering of the hypothalamic-pituitary-adrenocortical axis: A review of animal models and human studies across development. Psychological bulletin, 140(1), 256.

Kelly, M. M., Tyrka, A. R., Anderson, G. M., Price, L. H., \& Carpenter, L. L. (2008). Sex differences in emotional and physiological responses to the Trier Social Stress Test. Journal of behavior therapy and experimental psychiatry, 39(1), 87-98.

Kim, H. G., Cheon, E. J., Bai, D. S., Lee, Y. H., \& Koo, B. H. (2018). Stress and heart rate variability: a meta-analysis and review of the literature. Psychiatry investigation, 15(3), 235.

Kirschbaum, C. Why we need an online version of the Trier Social Stress Test. Psychoneuroendocrinology, 105129. 
ONLINE TSST ASSESSED BY PHOTOPLETHYSMOGRAPHY

Kirschbaum, C., Pirke, K. M., \& Hellhammer, D. H. (1993). The 'Trier Social Stress Test'-a tool for investigating psychobiological stress responses in a laboratory setting. Neuropsychobiology, 28(1-2), 76-81.

Lesage, F. X., Berjot, S., \& Deschamps, F. (2012). Clinical stress assessment using a visual analogue scale. Occupational medicine, 62(8), 600-605.

Lovallo, W. R., Cohoon, A. J., Acheson, A., Vincent, A. S., \& Sorocco, K. H. (2019). Cortisol stress reactivity in women, diurnal variations, and hormonal contraceptives: studies from the Family Health Patterns Project. Stress, 22(4), 421-427.

Nelson, B. W., Low, C. A., Jacobson, N., Areán, P., Torous, J., \& Allen, N. B. (2020). Guidelines for wrist-worn consumer wearable assessment of heart rate in biobehavioral research. NPJ Digital Medicine, 3(1), 1-9.

O'dea, S. (2020). Number of smartphone users worldwide from 2016 to 2021. Statista Research Department. https://www.statista.com/statistics/330695/number-of-smartphone-usersworldwide/

Riem, M. M., Kunst, L. E., Bekker, M. H., Fallon, M., \& Kupper, N. (2020). Intranasal oxytocin enhances stress-protective effects of social support in women with negative childhood experiences during a virtual Trier Social Stress Test. Psychoneuroendocrinology, 111, 104482.

Roos, L. E., Knight, E. L., Beauchamp, K. G., Giuliano, R. J., Fisher, P. A., \& Berkman, E. T. (2017). Conceptual precision is key in acute stress research: A commentary on Shields, Sazma, \& Yonelinas, 2016. Neuroscience \& Biobehavioral Reviews, 83, 140-144.

Sander, L., \& Bauman, O. (2020). 5 reasons why Zoom meetings are so exhausting. The Conversation, 5. https://theconversation.com/5-reasons-why-zoom-meetings-are-so- 
ONLINE TSST ASSESSED BY PHOTOPLETHYSMOGRAPHY

exhausting-137404

Shiban, Y., Diemer, J., Brandl, S., Zack, R., Mühlberger, A., \& Wüst, S. (2016). Trier Social Stress Test in vivo and in virtual reality: Dissociation of response domains. International Journal of Psychophysiology, 110, 47-55.

Spitzer, R. L., Kroenke, K., Williams, J. B., \& Löwe, B. (2006). A brief measure for assessing generalized anxiety disorder: the GAD-7. Archives of internal medicine, 166(10), 10921097.

Weiler, D. T., Villajuan, S. O., Edkins, L., Cleary, S., \& Saleem, J. J. (2017, September). Wearable heart rate monitor technology accuracy in research: a comparative study between PPG and ECG technology. In Proceedings of the Human Factors and Ergonomics Society Annual Meeting (Vol. 61, No. 1, pp. 1292-1296). Sage CA: Los Angeles, CA: SAGE Publications.

Von Dawans, B., Kirschbaum, C., \& Heinrichs, M. (2011). The Trier Social Stress Test for Groups (TSST-G): A new research tool for controlled simultaneous social stress exposure in a group format. Psychoneuroendocrinology, 36(4), 514-522. 
ONLINE TSST ASSESSED BY PHOTOPLETHYSMOGRAPHY

\section{Table 1}

Means, Standard Deviations and Pairwise Comparisons by Group on Heart Rate and Combined Stress and Anxiety measures

\begin{tabular}{|c|c|c|c|c|}
\hline & \multirow[t]{3}{*}{ Time } & \multicolumn{2}{|c|}{ Group } & \multirow[t]{3}{*}{ Pairwise Comparisons by Group } \\
\hline & & Control $(n=50)$ & Stress $(n=54)$ & \\
\hline & & $M(S D)$ & $M(S D)$ & \\
\hline Heart & 1 & $74.840(13.774)$ & $78.150(14.917)$ & $F(1,101)=1.324, p=.253$ \\
\hline \multirow[t]{6}{*}{ Rate } & 2 & $74.280(11.573)$ & $75.982(14.083)$ & $F(1,101)=.427, p=.515$ \\
\hline & 3 & $72.574(11.130)$ & $78.154(11.017)$ & $F(1,101)=6.468, p=.013^{*}$ \\
\hline & 4 & $73.811(13.892)$ & $83.068(15.428)$ & $F(1,101)=10.184, p=.002 * *$ \\
\hline & 5 & $74.603(12.123)$ & $81.795(15.792)$ & $F(1,101)=6.652, p=.010^{*}$ \\
\hline & 6 & $72.480(10.816)$ & $76.482(12.528)$ & $F(1,101)=2.610, p=.089$ \\
\hline & 7 & $70.460(9.272)$ & $75.890(11.495)$ & $F(1,101)=5.891, p=.009 * *$ \\
\hline Stress & 1 & $33.270(23.318)$ & $33.907(24.285)$ & $F(1,101)=.030, p=.863$ \\
\hline$\&$ & 2 & $26.132(22.604)$ & $28.328(23.368)$ & $F(1,101)=.259, p=.612$ \\
\hline \multirow[t]{5}{*}{ Anxiety } & 3 & $24.306(20.588)$ & $26.709(22.072)$ & $F(1,101)=.388, p=.535$ \\
\hline & 4 & $42.514(26.041)$ & $59.329(25.764)$ & $F(1,101)=12.846, p=.001 * *$ \\
\hline & 5 & $37.006(27.979)$ & $56.057(28.167)$ & $F(1,101)=13.552, p<.001 * * *$ \\
\hline & 6 & $21.770(20.284)$ & $31.538(24.385)$ & $F(1,101)=5.314, p=.023^{*}$ \\
\hline & 7 & $20.120(20.555)$ & $28.602(22.895)$ & $F(1,101)=4.349, p=.040^{*}$ \\
\hline
\end{tabular}

Note. Stress \& Anxiety = average score of self-reported stress and anxiety scores on a scale of 0 to $100 . * p<.05, * * p<.01, * * * p<.001$. 
ONLINE TSST ASSESSED BY PHOTOPLETHYSMOGRAPHY

\section{Table 2}

Results of a 2 (Group) 77 (Time) ANCOVA Examining Heart Rate and Self-reported Stress \& Anxiety While Controlling for Gender.

\begin{tabular}{|c|c|c|c|c|c|c|}
\hline \multirow[b]{2}{*}{ Source } & \multicolumn{3}{|c|}{ Heart Rate } & \multicolumn{3}{|c|}{ Stress \& Anxiety } \\
\hline & $d f$ & $F$ & $p$ & $d f$ & $F$ & $p$ \\
\hline \multicolumn{7}{|c|}{ Between-subjects effects } \\
\hline Gender & 1 & $<.001$ & .994 & 1 & 5.440 & $.022 *$ \\
\hline Group & 1 & 6.491 & $.012^{*}$ & 1 & 5.089 & $.026^{*}$ \\
\hline Error (Gender) & 101 & & & 101 & & \\
\hline \multicolumn{7}{|c|}{ Within-subjects effects } \\
\hline Time & 4.778 & .945 & .300 & 3.108 & 1.463 & .223 \\
\hline Time x Gender & 4.778 & .765 & .343 & 3.108 & 3.699 & $.011^{*}$ \\
\hline Time x Group & 4.778 & 2.449 & $.035^{*}$ & 3.108 & 7.960 & $<.001 * * *$ \\
\hline Error (time) & 482.588 & & & 313.915 & & \\
\hline
\end{tabular}

Note. $\mathrm{N}=104 . * p<.05, * * p<.01, * * * p<.001$. Greenhouse-Geisser adjusted degrees of freedom are reported for all within-subject effects. 
ONLINE TSST ASSESSED BY PHOTOPLETHYSMOGRAPHY

\section{Table 3}

P-values for Pairwise Comparisons of Visual Analogue Scale (VAS) Self-Reports of Stress \& Anxiety by Timepoint for the Stress and Control Groups.

Group

$$
\text { VAS1 VAS2 VAS3 VAS4 VAS5 VAS6 VAS7 }
$$

\begin{tabular}{|c|c|c|c|c|c|c|c|c|c|}
\hline Control & VAS1 & - & - & - & - & & - & - & - \\
\hline & VAS2 & $.001 * *$ & - & - & - & & - & - & - \\
\hline & VAS3 & $<.001^{* * *}$ & $*>.99$ & $9-$ & - & & - & - & - \\
\hline & VAS4 & .081 & $<.001 *$ & $* * *<$. & $.001 * * *$ & & - & - & - \\
\hline & VAS5 & $>.999$ & .113 & .01 & $11 \quad .576$ & & - & - & - \\
\hline & VAS6 & $<.001 * *$ & $*>.99$ & & $999<.001 *$ & $* * *<$ & $<.001^{* * * *}$ & * - & - \\
\hline & VAS7 & $<.001 * *$ & $* .175$ & .378 & $8<.001^{* * *}$ & & $001 * * *$ & $>.999$ & - \\
\hline Stress & VAS1 & - & - & - & - & - & - & - & \\
\hline & VAS2 & .999 & - & - & - & - & - & - & \\
\hline & VAS3 & .999 & $>.999$ & - & - & - & - & - & \\
\hline & VAS4 & .347 & $.003 * *$ & $026^{*}$ & - & - & - & - & \\
\hline & VAS5 & $<.001 * *$ & & .077 & $.283>$. & .999 & - & - & 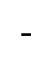 \\
\hline & VAS6 & $>.999>$ & $>.999$ & $>.999$ & $<.001^{* * *}$ & $.002^{*}$ & ** & - & \\
\hline & VAS7 & .540 & $>.999$ & $>.999$ & $.001^{* *}$ & $.003 *$ & $* *>.999$ & - & \\
\hline
\end{tabular}

Note. VAS = visual analogue scale self-reports of Stress and Anxiety at each of the seven timeopints. $* p<.05, * * p<.01, * * * p<.001$. 
ONLINE TSST ASSESSED BY PHOTOPLETHYSMOGRAPHY

\section{Table 4}

P-values for Pairwise Comparisons of Heart Rate (HR) by Timepoint for the Stress and Control Groups.

Group

HR1 HR2 HR3 HR4 HR5 HR6 HR7

\begin{tabular}{|c|c|c|c|c|c|c|c|c|}
\hline Control & HR1 & - & - & - & - & - & - & - \\
\hline & HR2 & $>.999$ & - & - & - & - & - & - \\
\hline & HR3 & $>.999$ & $>.999$ & - & - & - & - & - \\
\hline & HR4 & $>.999$ & $>.999$ & $>.999$ & - & - & - & - \\
\hline & HR5 & $>.999$ & $>.999$ & $>.999$ & $>.999$ & - & - & - \\
\hline & HR6 & $>.999$ & $>.999$ & $>.999$ & $>.999$ & $>.999$ & - & - \\
\hline & HR7 & .235 & .287 & $>.999$ & $>.999$ & .183 & $>.999$ & - \\
\hline Stress & HR1 & - & - & - & - & - & - & - \\
\hline & HR2 & $>.999$ & - & - & - & - & - & - \\
\hline & HR3 & $>.999$ & $>.999$ & - & - & - & - & - \\
\hline & HR4 & .347 & $.003 * *$ & $.026^{*}$ & - & - & - & - \\
\hline & HR5 & $>.999$ & .077 & .283 & $>.999$ & - & - & - \\
\hline & HR6 & $>.999$ & $>.999$ & $>.999$ & $<.001 * * *$ & *.002* & k* - & - \\
\hline & HR7 & $>.999$ & $>.999$ & $>.999$ & $.001 * *$ & $.003 *$ & $* *>999$ & - \\
\hline
\end{tabular}

Note. Bonferroni-corrections were applied for multiple comparisons. ${ }^{*} p<.05, * * p<.01, * * * p$ $<.001$. 
ONLINE TSST ASSESSED BY PHOTOPLETHYSMOGRAPHY

Table 5

Results from Independent Sample t-tests Examining Gender Differences in Heart Rate and Selfreported Stress \& Anxiety Measures for the Stress and Control Groups at Each Timepoint.

\begin{tabular}{|c|c|c|c|c|c|c|c|}
\hline \multirow[t]{2}{*}{ Measure } & \multirow[t]{2}{*}{ Timepoint } & \multicolumn{3}{|c|}{ Control $(n=49)$} & \multicolumn{3}{|c|}{ Stress $(n=53)$} \\
\hline & & $M(S D)$ & $t$ & $p$ & $M(S D)$ & $t$ & $p$ \\
\hline \multirow[t]{7}{*}{ Heart Rate } & 1 & $\begin{array}{l}3.912 \\
(4.181)\end{array}$ & 0.936 & 0.354 & $\begin{array}{l}-0.716 \\
(4.290)\end{array}$ & -0.167 & 0.868 \\
\hline & 2 & $\begin{array}{l}2.345 \\
(3.529)\end{array}$ & 0.665 & 0.509 & $\begin{array}{l}-0.040 \\
(4.051)\end{array}$ & -0.010 & 0.992 \\
\hline & 3 & $\begin{array}{l}2.897 \\
(3.384)\end{array}$ & 0.856 & 0.396 & $\begin{array}{l}-0.968 \\
(3.114)\end{array}$ & -0.306 & 0.761 \\
\hline & 4 & $\begin{array}{l}1.339 \\
(4.251)\end{array}$ & 0.315 & 0.754 & $\begin{array}{l}-1.590 \\
(4.433)\end{array}$ & -0.359 & 0.721 \\
\hline & 5 & $\begin{array}{l}-1.962 \\
(3.702)\end{array}$ & -0.530 & 0.599 & $\begin{array}{l}-2.782 \\
(4.527)\end{array}$ & -0.615 & 0.541 \\
\hline & 6 & $\begin{array}{l}1.275 \\
(3.308)\end{array}$ & 0.385 & 0.702 & $\begin{array}{l}0.483 \\
(3.604)\end{array}$ & 0.134 & 0.894 \\
\hline & 7 & $\begin{array}{l}-1.228 \\
(2.835)\end{array}$ & -0.433 & 0.667 & $\begin{array}{l}-2.183 \\
(3.293)\end{array}$ & -0.633 & 0.510 \\
\hline \multirow[t]{7}{*}{$\begin{array}{l}\text { Stress \& } \\
\text { Anxiety }\end{array}$} & 1 & $\begin{array}{l}-6.601 \\
(7.079)\end{array}$ & -0.933 & 0.356 & $\begin{array}{l}-4.323 \\
(6.961)\end{array}$ & -0.621 & 0.537 \\
\hline & 2 & $\begin{array}{l}-3.636 \\
(6.904)\end{array}$ & -0.527 & 0.601 & $\begin{array}{l}-3.262 \\
(6.707)\end{array}$ & -0.486 & 0.629 \\
\hline & 3 & $\begin{array}{l}-8.905 \\
(6.174)\end{array}$ & -1.442 & 0.156 & $\begin{array}{l}-3.901 \\
(6.327)\end{array}$ & -0.617 & 0.540 \\
\hline & 4 & $\begin{array}{l}-16.509 \\
(7.612)\end{array}$ & -2.169 & $0.035 *$ & $\begin{array}{c}-17.962 \\
(6.981)\end{array}$ & -2.573 & $0.013 *$ \\
\hline & 5 & $\begin{array}{l}-18.171 \\
(8.159)\end{array}$ & -2.227 & $0.031 *$ & $\begin{array}{l}-15.070 \\
(7.829)\end{array}$ & -1.925 & 0.060 \\
\hline & 6 & $\begin{array}{l}-8.164 \\
(6.100)\end{array}$ & -1.338 & 0.187 & $\begin{array}{l}-9.647 \\
(6.887)\end{array}$ & -1.401 & 0.167 \\
\hline & 7 & $\begin{array}{l}-8.587 \\
(6.173)\end{array}$ & -1.391 & 0.171 & $\begin{array}{l}-.10 .104 \\
(6.436)\end{array}$ & -1.570 & 0.122 \\
\hline
\end{tabular}

Note. $M=$ Mean difference, $S D=$ Standard Error Difference. $* \mathrm{p}<.05$. 
ONLINE TSST ASSESSED BY PHOTOPLETHYSMOGRAPHY

\section{Figure 1}

Line Graphs Depicting Measured Heart Rate and Self-Reported Stress \& Anxiety Throughout the Experiment.
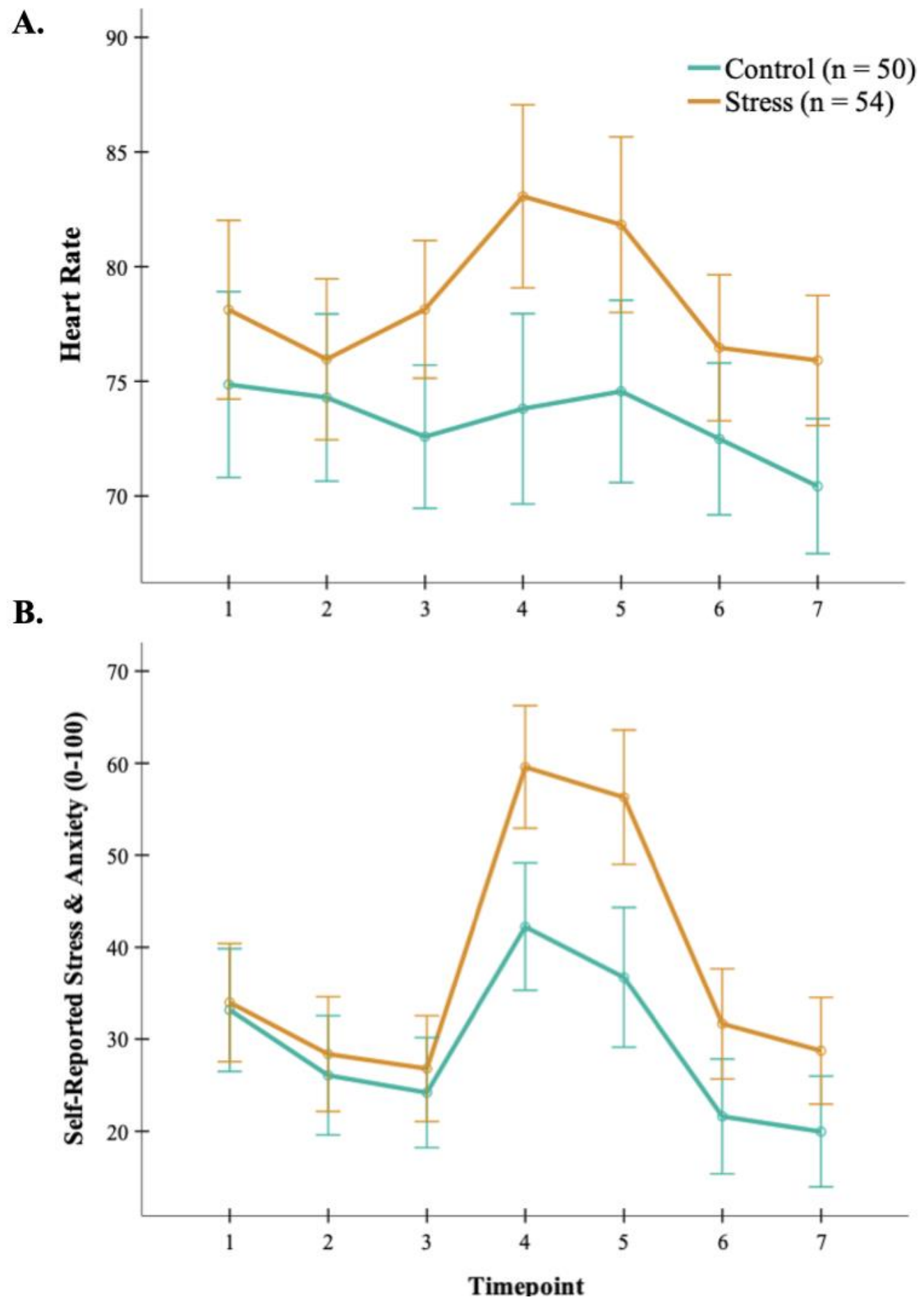

Note. Error Bars reflect $95 \%$ confidence intervals. 
ONLINE TSST ASSESSED BY PHOTOPLETHYSMOGRAPHY

Figure 2

Raincloud Plots Depicting Individual Data Points, Box Plots, and Histograms for Measured Heart Rate and Self-reported Stress \& Anxiety Throughout the Experiment.

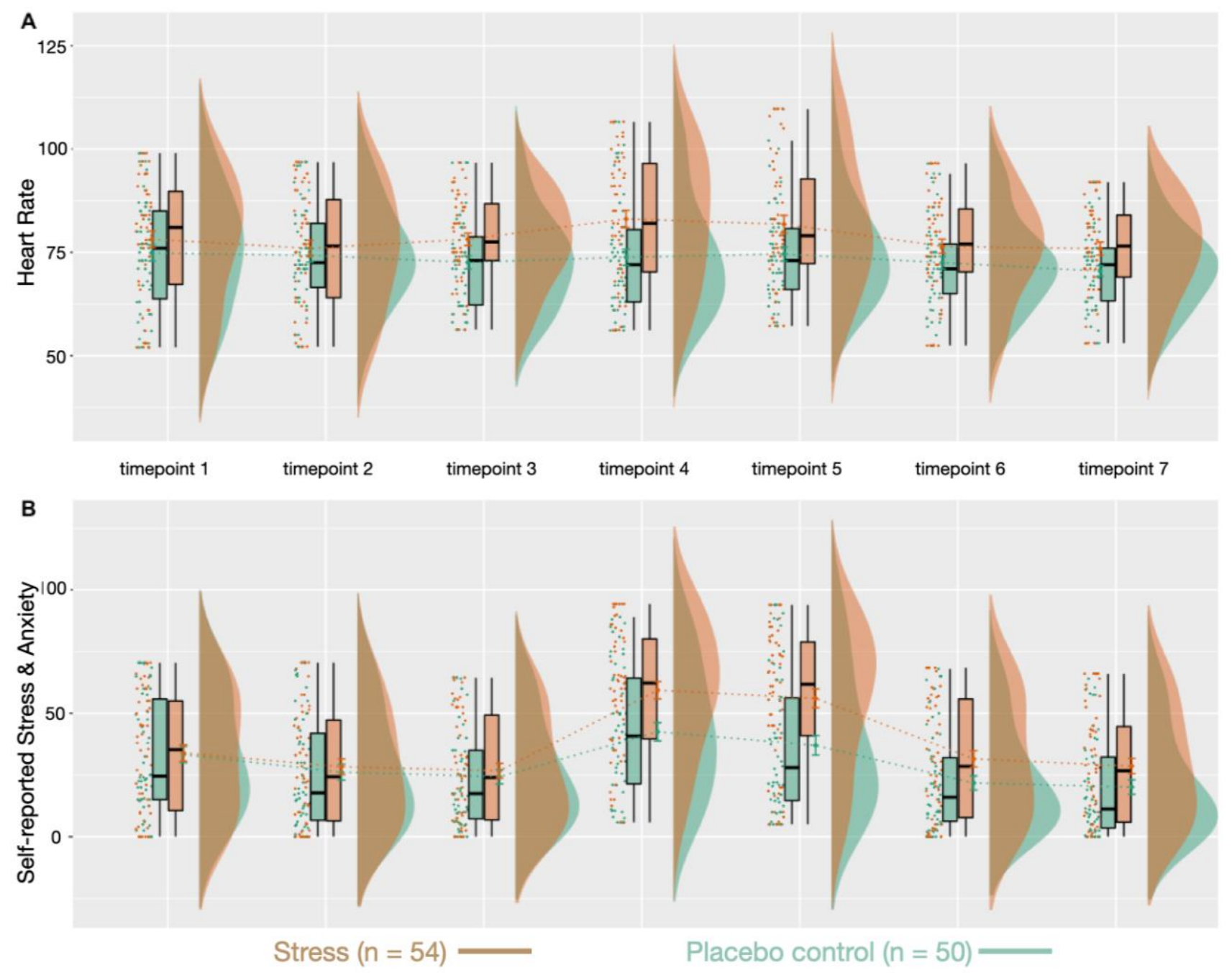

